\title{
Genome-wide association study of the five-factor model of personality in young Korean women
}

Han-Na Kim, Seung-Ju Roh, Yeon Ah Sung, Hye Won Chung, Jong-Young Lee, Juhee Cho, Hocheol Shin and Hyung-Lae Kim

Journal of Human Genetics (2015) 60, 463; doi:10.1038/jhg.2015.67

Correction to: Journal of Human Genetics (2013) 58, 667-674; doi:10.1038/jhg.2013.75; published online 1 August 2013

The authors of the above paper noticed errors in publication. OR1A2 in the abstract, keywords and discussion was incorrectly given as ORIA2. This correction does not alter the results and their interpretation as discussed in the paper.

The authors would like to apologize for these mistakes. 